\title{
Ultrasound-Guided Transbronchial Needle-Delivered Interstitial Photodynamic Therapy
}

National Cancer Institute

\section{Source}

National Cancer Institute. Ultrasound-Guided Transbronchial Needle-Delivered Interstitial Photodynamic Therapy. NCI Thesaurus. Code C155857.

Photodynamic treatment of deep-seated solid tumors using a light source that is placed by a transbronchial needle, using ultrasound guidance. 